\title{
Acetylcholinesterase Inhibitors in Myasthenic Crisis: A Systematic Review of Observational Studies
}

\author{
Mario B. Prado Jr. ${ }^{1,2^{*}}$ and Karen Joy Adiao ${ }^{3}$ \\ @ 2021 Springer Science+Business Media, LLC, part of Springer Nature and Neurocritical Care Society
}

\begin{abstract}
Current myasthenia gravis guidelines recommend intravenous immunoglobulin or plasmapheresis and discontinuation of pyridostigmine during myasthenic crisis. However, intravenous immunoglobulin or plasmapheresis is expensive and frequently not available in developing countries. This study aims to summarize the evidence of giving an acetylcholinesterase inhibitor in myasthenic crisis. Medline, Embase, and Cochrane databases and references were searched for observational studies that determined the use of acetylcholinesterase inhibitor in myasthenic crisis. The eligibility criteria were as follows: population, patients with myasthenic crisis, intervention (acetylcholinesterase inhibitor administration), and outcome (clinical improvement and complications). In total, 106 studies were identified, 92 through database searching (after removing duplicates) and 14 through other sources. Only eight were analyzed in the present systematic review. In five, acetylcholinesterase inhibitor was given at the start of the crisis, whereas in the other three, acetylcholinesterase inhibitor was discontinued initially and then restarted prior to extubation. Two observational analytic studies and three case reports showed improvement in different outcome measures. In the other three, improvement of outcome measures was also observed. Overall, a small proportion of patients developed cardiac arrhythmia and pneumonia after administration of acetylcholinesterase inhibitor alone, although this was not statistically different compared with those subjected to plasmapheresis. In summary, continuous intravenous infusion of pyridostigmine or neostigmine can be a substitute for intravenous immunoglobulin or plasmapheresis if these are not available during crisis; however, caution should be observed because of the aforementioned possible complications.
\end{abstract}

Keywords: Myasthenic crisis, Acetylcholinesterase inhibitor, Pyridostigmine, Myasthenia gravis

\section{Introduction}

Myasthenia gravis (MG) is a rare autoimmune condition of the neuromuscular junction characterized by fluctuating muscle weakness [1]. Frequently, it affects the eye and bulbar muscles and rarely the limbs [2]. Because of advancement in the diagnosis and treatment, the mortality rate has declined from 40 to $5 \%$ even in worst conditions [3]. Most of the time, MG symptoms are

\footnotetext{
*Correspondence: mbprado@alum.up.edu.ph

1 Department of Neurology, Graduate School of Medicine, Chiba University, 1-8-1 Inohana, Chuo-ku, Chiba 260-8670, Japan Full list of author information is available at the end of the article
}

precipitated by infection, initiation of steroids, intake of medications that affect the neuromuscular junction and surgery [4].

Severe affection of the bulbar and accessory muscles and diaphragm that necessitates invasive or noninvasive ventilation is termed myasthenic crisis (MC) [3]. MC should be differentiated from cholinergic crisis, a condition with similar presentation, brought about by excessive intake of acetylcholinesterase inhibitor (AChEI). Although theoretically the management of $\mathrm{MC}$ and cholinergic crisis $(\mathrm{CC})$ differs, $\mathrm{CC}$ has rarely been diagnosed in any studies $[3,5,6]$.

\section{实


Once the respiration of a patient with $\mathrm{MC}$ has been stabilized, intravenous immunoglobulin (IVIG) or plasmapheresis (PLEx) with an addition of corticosteroids is administered [7]. Because of possible worsening from increased bronchial secretions, many review articles and guidelines suggest discontinuation of AChEI during the acute period of MC [8]. However, these recommendations were based on few observational studies with a limited number of subjects. Conversely, earlier studies suggest that administration of AChEI may actually complement IVIG, PLEx, and corticosteroids in improving the condition $[6,9]$.

PLEx and IVIG are the first-line treatments for MC [10]. However, aside from the their cost and side effects, technical skills for their administration and dependence to machines for PLEx limit their use in developing countries [1]. This study determines the evidence of AChEI in patients with $\mathrm{MC}$ in improving the outcome of this condition in the setting where PLEx and IVIG are not available.

\section{Objectives}

This study aims to determine the utility of AChEI in patients with $\mathrm{MC}$ by collecting all the possible available evidence for its use. Specifically, it aims to answer the following questions:

1. Can we use AChEI in MC?

2. If yes, what type(s) of AChEI can be given?

3. What is the preferred route of administration and dosage?

4. When is the best time to give AChEI in patients with $\mathrm{MC}$ ?

5. What is the effect of adding AChEI to existing recommended first-line medications or management for $\mathrm{MC}$ ?

6. What are the side effects/complications associated with AChEI?

7. Is AChEI safe when given alone during $\mathrm{MC}$ ?

\section{Methodology}

\section{Protocol Registration}

Upon searching Cochrane and Prospero Registered Systematic Reviews, no existing reviews of the same objectives were found.

\section{Eligibility Criteria}

Only studies fulfilling the following eligibility criteria were included.

\section{Study Population}

Studies in which the inclusion criteria contained patients with MC or Myasthenia Gravis Foundation of
America (MGFA) 5 treated with AChEI alone or in combination with other treatment options during the crisis period were selected.

\section{Type of Exposure or Intervention Used}

Studies wherein AChEI was given during intubation or restarted prior to extubation were included in the analysis. The routes of administration included intravenous (IV), intramuscular (IM), and oral forms of AChEI, combined with the standard of care treatment for patients in MC (i.e., IVIG, Total Plasma Exchange, and high-dose corticosteroids, or combination thereof). Any studies in which AChEI in MC has been analyzed as a secondary objective were also considered.

\section{Type of Studies}

Because studies about the utility of AChEI in MC were sparse, case reports, case series, case-control studies, and retrospective and prospective cohort and nonrandomized trials were included. Further, including nonrandomized trials made the search more comprehensive and generalizable. Published peer-reviewed journals were given priority, although other related unpublished literatures were also considered to decrease publication bias. All studies from 1963 to 2020 were scanned and considered to increase the sensitivity of the search. English written journals and English-translated journals were considered.

\section{Information Sources}

The following databases and registries were searched:

- Pubmed/Medline (1963 to 2020)

- Cochrane Central/Review (2002 to 2020)

- Embase

- Trial registers and clinical study reports (Clincaltrials.gov and World Health Organization International Clinical trials Registry Platform; no result)

Peer-reviewed journals were searched for studies fulfilling the eligibility criteria. Authors of studies were emailed to gather more information if needed. The primary authors (MBP and KJA) conducted search of these databases. Ongoing and unpublished data sources were also searched whenever possible by using RIAT.

\section{Search Process}

Two review authors (MBP and KJA), both neurologists, conducted the search and selected the journals. Whenever there was disagreement, a third-party consultant (KP) was involved in the decision-making process. The present systematic review, guided by the Meta-Analyses of Observational Studies in Epidemiology guidelines [11], 
was based on our reading of the standard medical texts and search of Cochrane, PubMed Medline, and Embase for articles from 1963 to 2020 using the terms ((()myasthenic crisis) OR (MGFA V))) AND ((((Acetylcholinesterase inhibitor) OR (pyridostigmine)) OR (pyridostigmine bromide)))). The search strategy terms were approved by authors and was done in September 2020. No additional filters were included in the search.

\section{Data Extraction and Management}

Both review authors independently extracted data from eligible studies using a pretested data collection form. Data collected include name of the author; year published; country; study design; duration of data collection; the baseline characteristics of the population, including the mean age, sex, duration of the symptoms, mean time to crisis, and precipitating factors; the participants, outcome measures, results, and conclusions. The collated data are summarized in Table 1. Excluded articles and reasons of exclusion are summarized in Table 2.

Because of the heterogeneity and lack of consensus for the application of risk assessment tools for observational studies, assessment of bias was not done. Nevertheless, observational studies were considered as biased and subject to the effect of confounders.

\section{Results}

\section{Result of the Search}

In total, 111 studies were identified, 97 through database searching (Medline: 93, Cochrane: 4) and 14 through other sources (see Fig. 1). After duplicated records were removed, only 106 were screened based on titles and abstracts. Whenever full articles were not available, and the titles and abstracts were deemed irrelevant to the present systematic review, they were excluded. In total, 79 records were excluded. The full articles of the 27 journals were retrieved and assessed for eligibility. Although all articles came from different countries, such as India, USA, England, and other European countries, all were written in English. Some authors were emailed to request for the full article, but without reply. Only eight journals satisfied the eligibility criteria and were included in the present review. The search had $100 \%$ sensitivity and $29.6 \%$ precision.

\section{Excluded Articles}

Of the 27 articles initially deemed eligible, 19 were excluded. Ten were review articles, four did not fulfil the eligibility criteria, three had no abstract and full articles, and two were letters to the editor (see Table 2).

\section{Studies Included}

Summaries of the characteristics of all the included studies were tabulated (see Table 1). The eight studies spanned from 1983 to 2013 and were conducted in the USA (3), India (3), Greece (1), and Germany (1). There were three case reports and three retrospective and two prospective case reviews.

\section{Participants}

Patient enrollment spanned from 1970 to 2010 and amassed 445 patients with MG, 183 of whom had 189 episodes of MC. The median age of patients who had MC was 41.5 years (range 10 to 65 years), and the median interval from onset of symptoms to first crisis was 8 months. The most common triggers were infections, cholinesterase withdrawal, thymectomy, and steroid initiation. All eight studies included patients with MC. Among retrospective and prospective case studies, one limited the subjects to older patients only. Among the case reports, two patients had postthymectomy crisis.

\section{Types of Intervention}

AChEI was given during $\mathrm{MC}$ in all studies. In five studies, AChEI was given at the start of the MC, whereas the other three studies discontinued AChEI initially and restarted it prior to extubation or near discharge. The five studies that gave AChEI during the onset of MC, used either pyridostigmine (3) or neostigmine (2) via continuous IV infusion (3), IM (1), or oral (1) routes. The dose for continuous IV infusion of pyridostigmine was $2-4 \mathrm{mg} / \mathrm{hr}$ titrated by $0.5 \mathrm{mg} / \mathrm{hr}$ to $1.0 \mathrm{mg} / \mathrm{hr}$ increments, whereas for continuous IV infusion of neostigmine, a dose was 0.002 to $0.012 \mathrm{mg} / \mathrm{kg} / \mathrm{hr}$ titrated using $0.002-\mathrm{mg} / \mathrm{kg} / \mathrm{hr}$ increments. The dose for the IM route was $1 \mathrm{mg}$ every $6 \mathrm{~h} \mathrm{[12].} \mathrm{In} \mathrm{the} \mathrm{case} \mathrm{report} \mathrm{that} \mathrm{used} \mathrm{oral} \mathrm{pyridostigmine,}$ dose as high as $120 \mathrm{mg}$ every $4 \mathrm{~h}$, combined with intermittent noninvasive ventilation, was given [13]. In studies that discontinued AChEI initially, low-dose oral pyridostigmine was started.

In all studies, there was cointervention of methylprednisolone pulse therapy (MPPT), IVIG, PLEx, azathioprine, or a combination of these with AChEI, except for one study in which the effect of IV pyridostigmine was compared with that of pyridostigmine plus prednisone and PLEx cohorts to given.

\section{Types of Outcomes}

Six studies assessed for incidence of complications, and five studies assessed duration of MC with invasive or noninvasive ventilation. Five studies also used clinical 


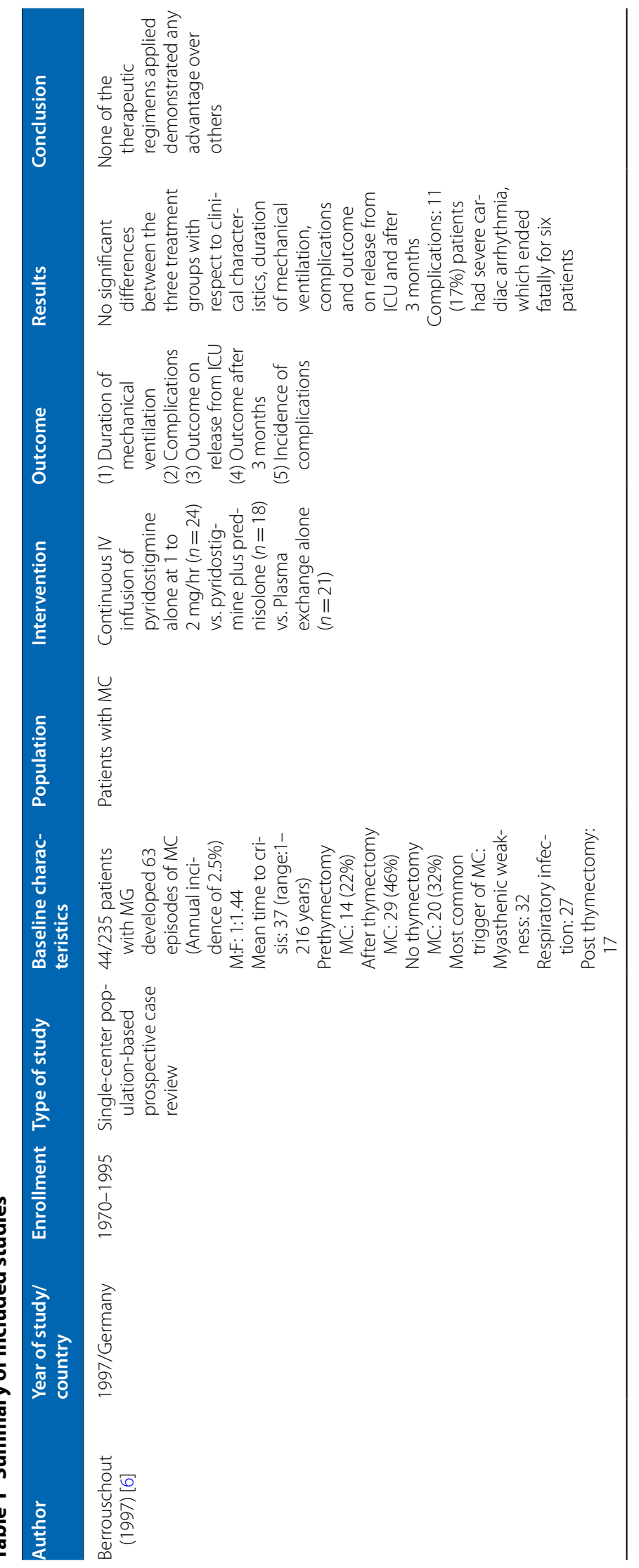




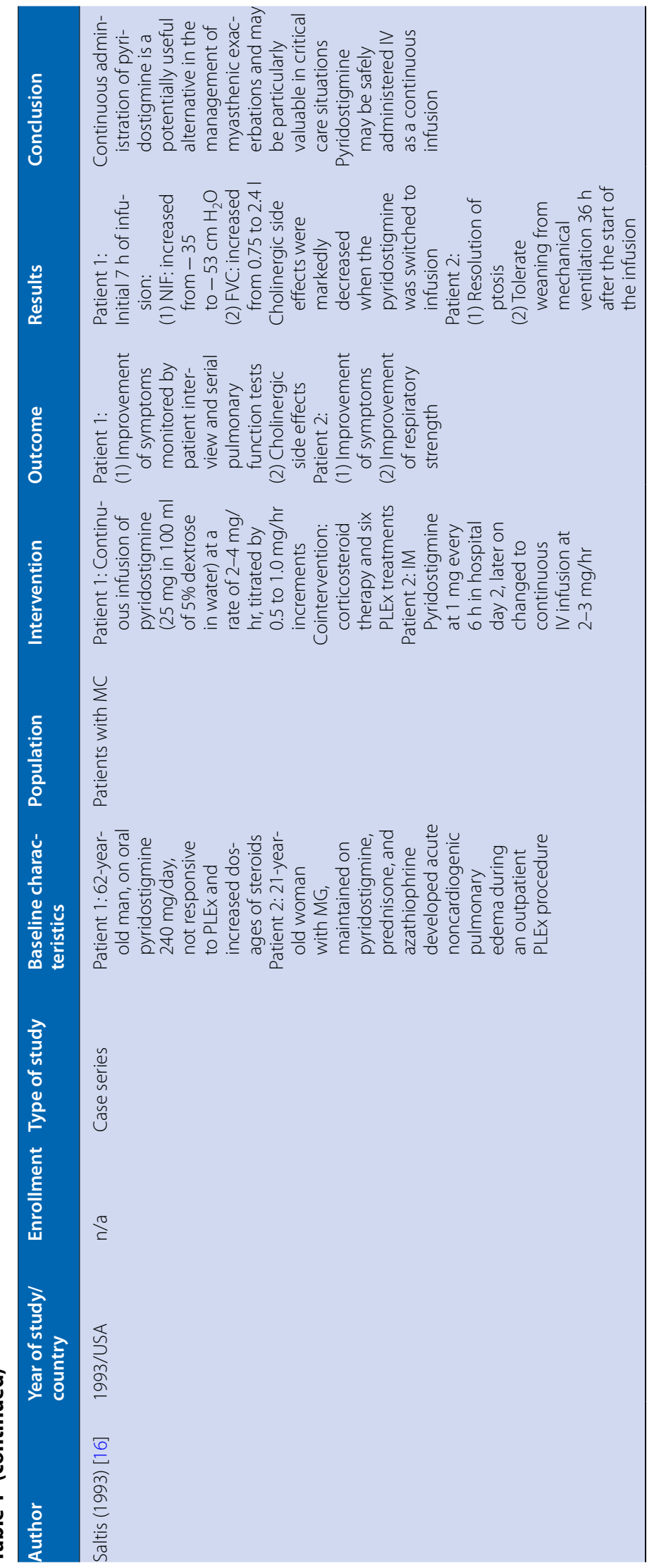




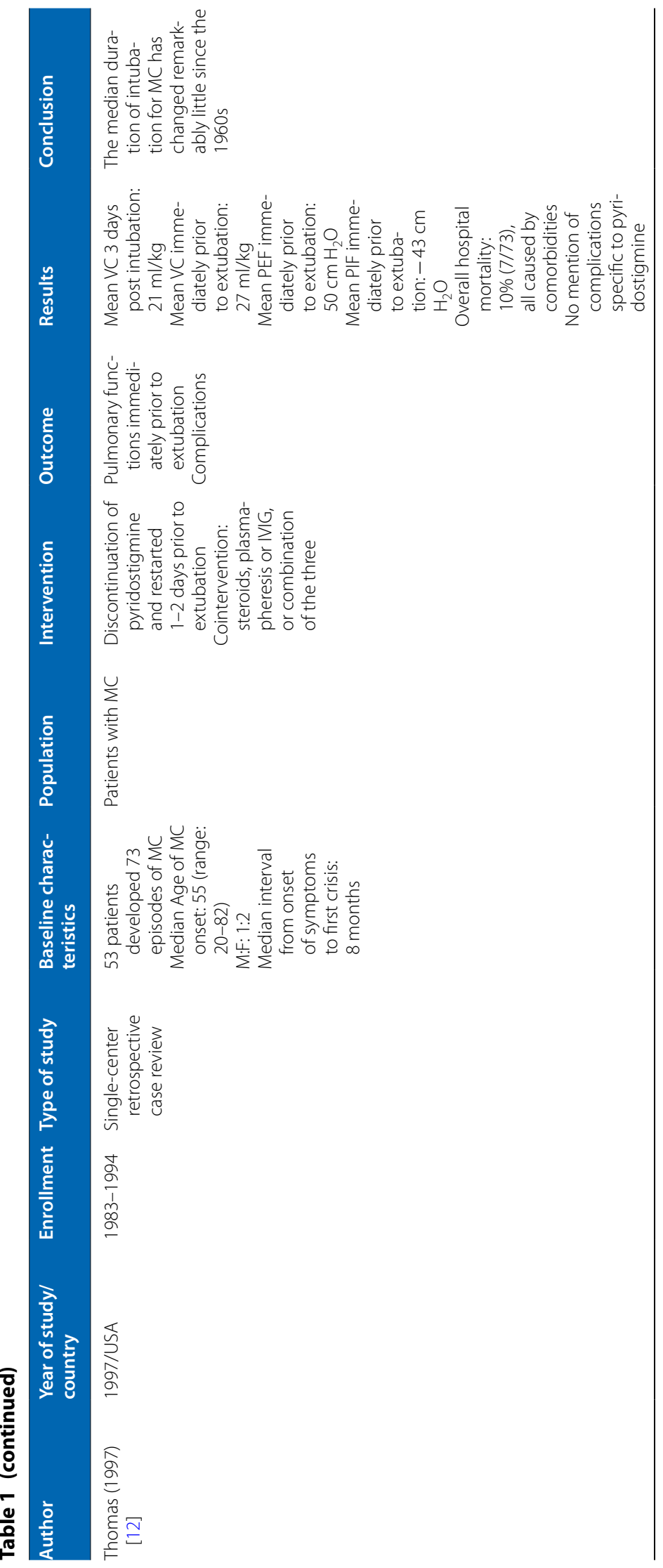




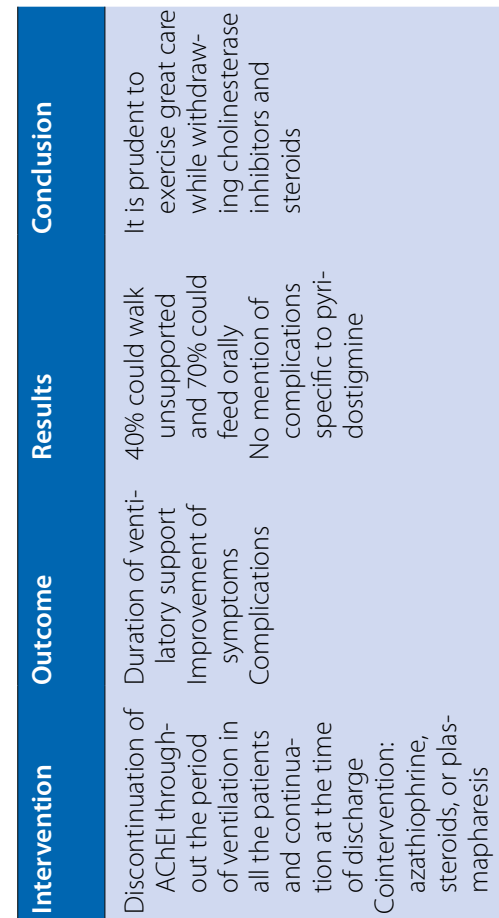

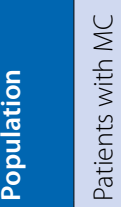

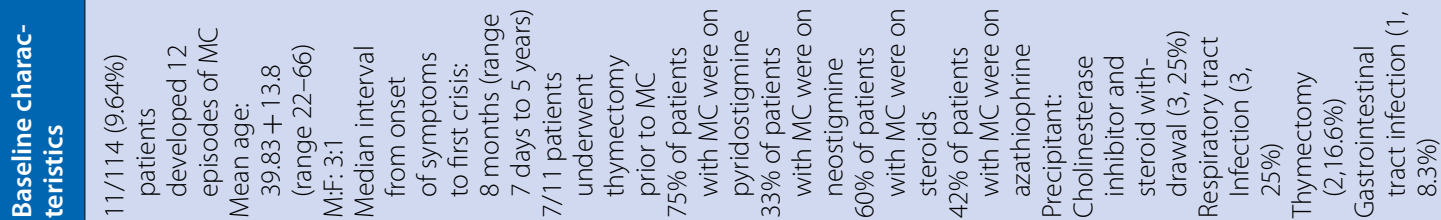

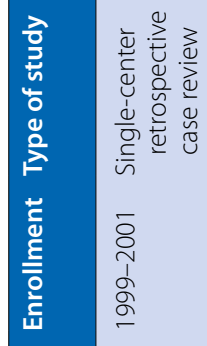

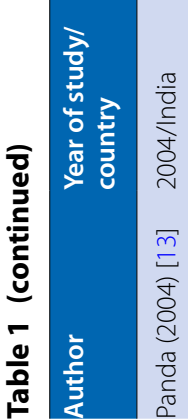




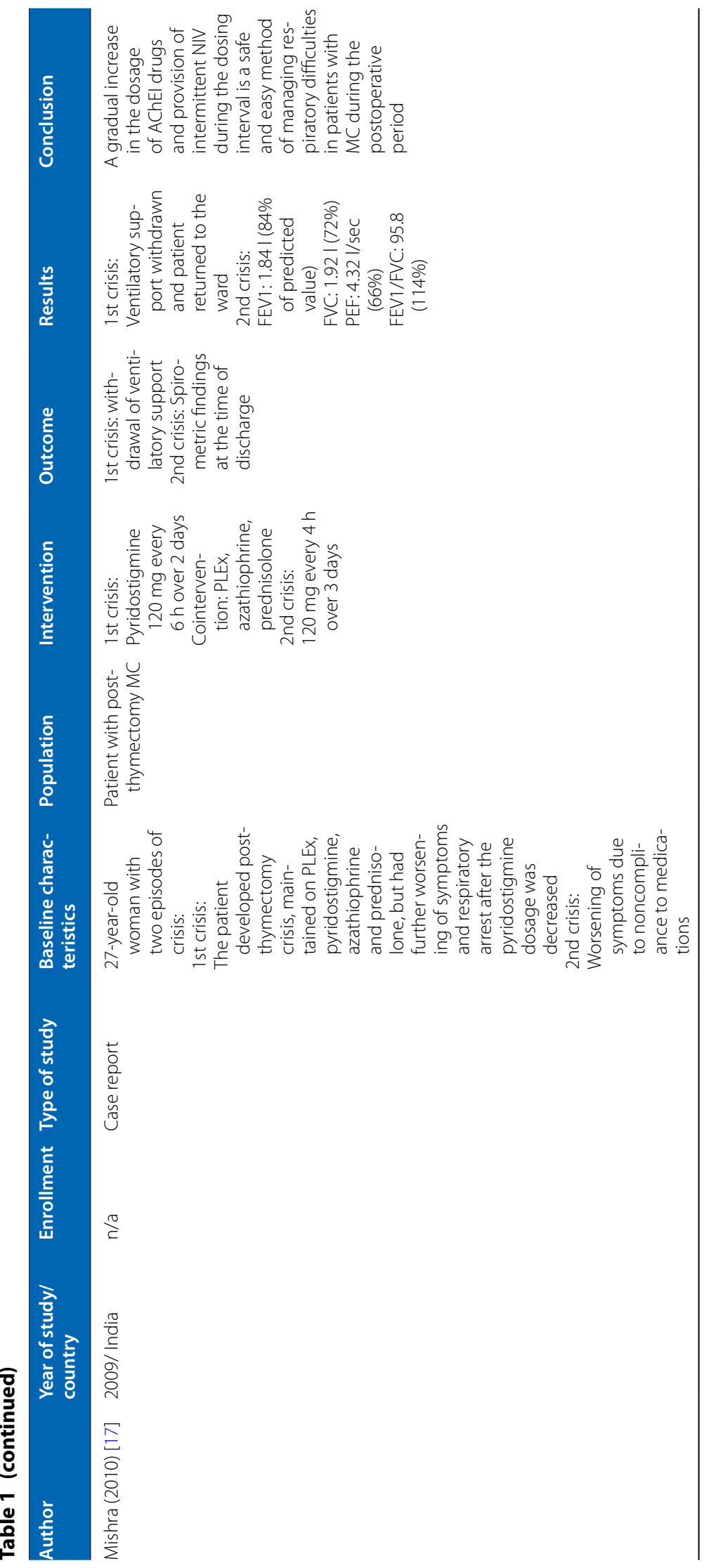




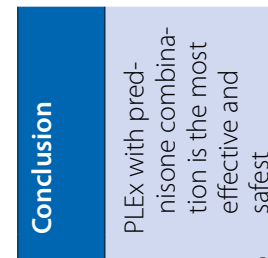

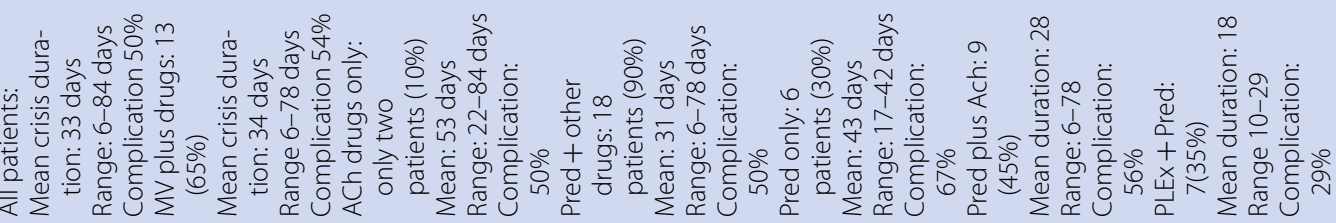

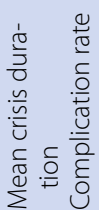

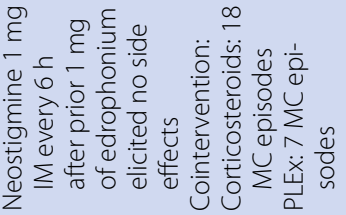

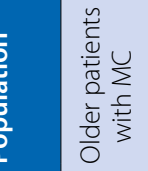

宛

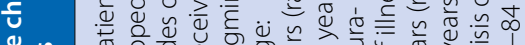

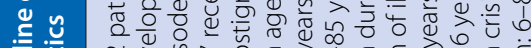

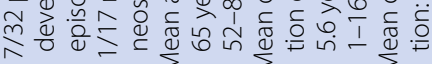

(1)

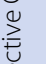

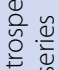

$\stackrel{2}{\gtrless}$

$\approx$

$\stackrel{\infty}{\circ}$

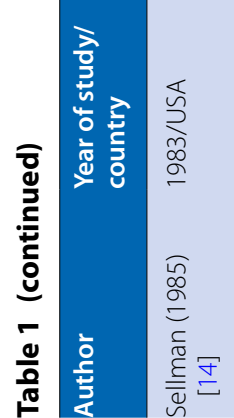




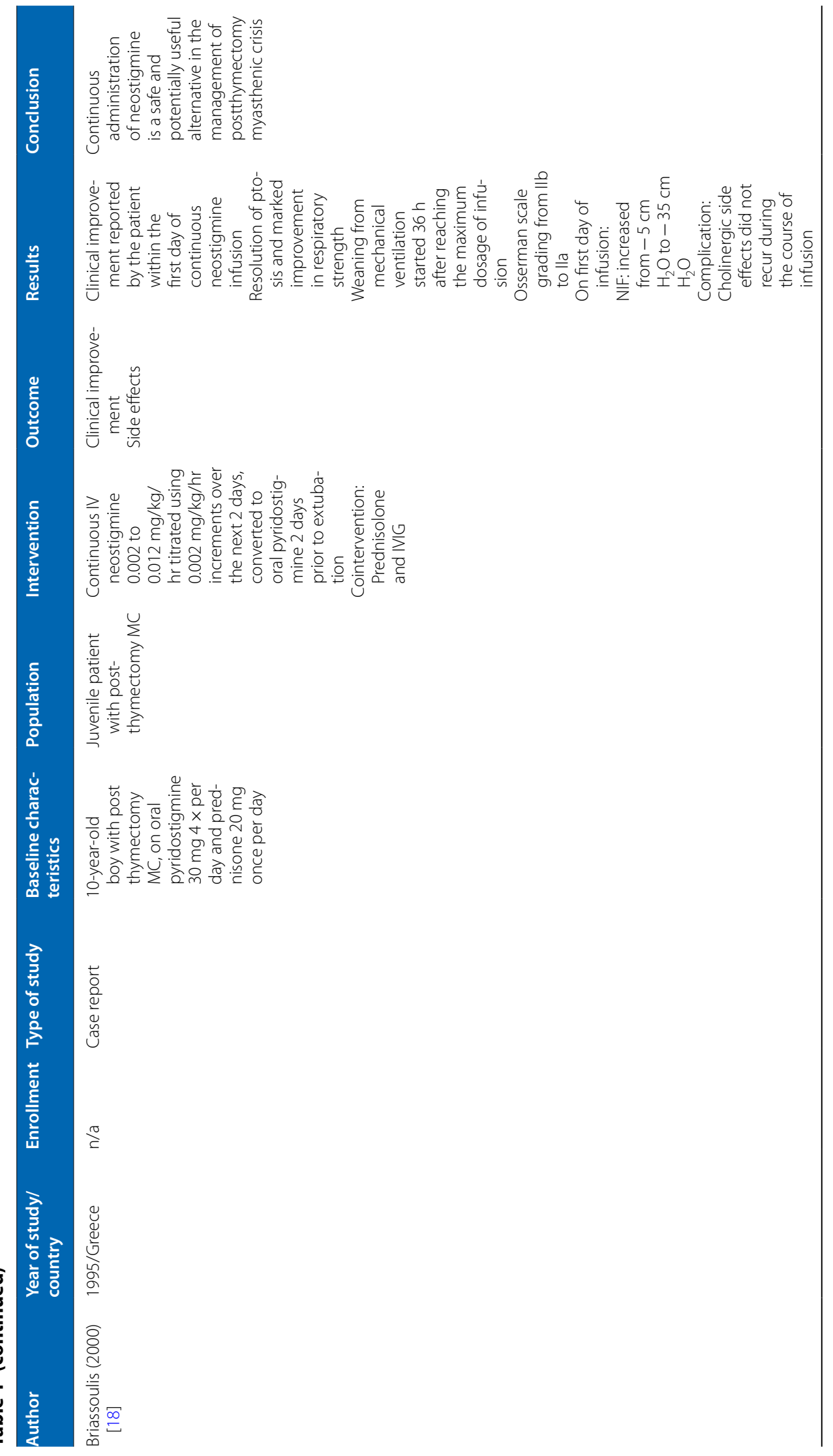




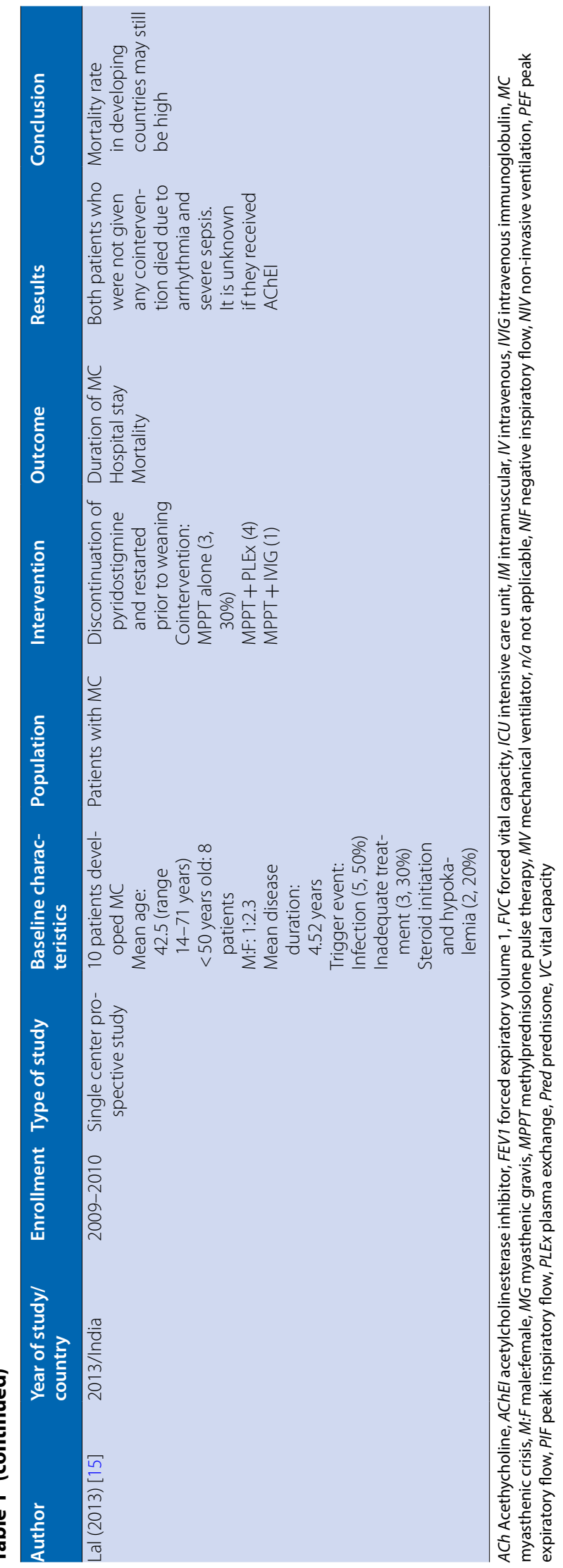


Table 2 Table of excluded studies

\begin{tabular}{|c|c|c|c|}
\hline Title & Author & Year & Reason for exclusion \\
\hline "Therapy for Myasthenic Crisis" & Mayer & 1998 & No abstract and full article \\
\hline "Continuous Infusion of Pyridostigmine for Myasthenic Crisis" & Nicholson & 1994 & No abstract and full article \\
\hline "Management of myasthenic crisis" & Kumar & 2005 & Letters \\
\hline "Myasthenic Crisis-Your Assessment Counts" & Hickey & 1991 & No abstract and full article \\
\hline $\begin{array}{l}\text { "Prescription Profile of Pyridostigmine in a Population of Patients with Myasthenia } \\
\text { Gravis" }\end{array}$ & Machado Alba & 2015 & Eligibility criteria for population not satisfied \\
\hline $\begin{array}{l}\text { "Administering Neostigmine as Subcutaneous Infusion: A Case Report of a Patient } \\
\text { Dying with Myasthenia Gravis" }\end{array}$ & Hindmarsh & 2019 & $\begin{array}{l}\text { Eligibility criteria for population not satisfied } \\
\text { Pop: MG with anal carcinoma }\end{array}$ \\
\hline $\begin{array}{l}\text { "Coronary Vasospasm Secondary to Hypercholinergic Crisis: An latrogenic Cause } \\
\text { of Acute Myocardial Infarction In Myasthenia Gravis" }\end{array}$ & Comerci & 2005 & $\begin{array}{l}\text { Eligibility criteria for population not fulfilled } \\
\text { Pop: patient with MG and MI }\end{array}$ \\
\hline "Myasthenia Gravis: Management of Myasthenic Crisis and Perioperative Period" & Juel & 2004 & Review article \\
\hline "An Update on Myasthenic Crisis" & Ahmed & 2005 & Review article \\
\hline "Myasthenic Crisis" & Lacomis & & Review article \\
\hline $\begin{array}{l}\text { "Approach to the Acute Management of Myasthenia Gravis and Guillain Barre } \\
\text { Syndrome" }\end{array}$ & Lizzariaga & 2016 & Review article \\
\hline "Myasthenic Crisis" & Kirmany & 2004 & Review article \\
\hline "Myasthenic Crisis: Guidelines for Prevention and Treatment" & Jani-Acsadi & 2007 & Review article \\
\hline "Myasthenic Crisis" & Wendell & 2011 & Review article \\
\hline $\begin{array}{l}\text { "The Patient in Myasthenic Crisis: An Update of the Management in Neurointen- } \\
\text { sive Care Unit" }\end{array}$ & Godoy & 2013 & Review article \\
\hline "Myasthenia Gravis Crisis" & Bershad & 2008 & Review article \\
\hline "Myasthenic Crisis" & Chaudhuri & 2008 & Review article \\
\hline $\begin{array}{l}\text { "Comparative Effects Of Plasma Exchange And Pyridostigmine on Respiratory } \\
\text { Muscle Strength and Breathing Pattern in Patients With Myasthenia Gravis" }\end{array}$ & Goti & 1995 & Eligibility criteria for population not fulfilled \\
\hline "Management of Myasthenic Crisis: Continuous Anticholinesterase Infusions" & Borel & 1993 & Letter to the editor \\
\hline
\end{tabular}

MG myasthenic gravis, Ml myocardial infarction, Pop population

improvement using serial pulmonary tests as outcome measures.

\section{Risk of Bias in Included Studies}

No bias assessment tool was employed in this systematic review. However, unlike in experimental studies, an exposure variable cannot be assigned to treatment groups in observational studies; hence, selection bias and confounding may be prevalent. In two studies, patients with infection and unstable hemodynamic status (cardiac problems) were given AChEI because these were contraindications to PLEx $[6,14]$. Consequently, the duration of the MC period as well as the complication rate will be longer and higher, respectively, in the AChEI only group. One of these studies employed elderly patients only; hence, this effect was magnified [14].

Cointervention with other medications confounds the effect of AChEI to MC outcome measures and side effects.

\section{Descriptive Analysis of the Studies}

1. Berrouschot et al. (1997) [6] conducted a singlecenter population-based prospective case review that included 44 patients with MG who developed 63 episodes of MC. This study compared three possible treatment options (AChEI only, AChEI + steroids, and PLEx) for $M C$ at that time. Patients with $M C$ exacerbated by respiratory infections and steroids were only given AChEI (continuous IV pyridostigmine, $n=24$ ), whereas those with no medical comorbidities and assumed to be primarily worsened by a pure myasthenic state were treated with a combination of AChEI + steroids $(n=18)$ or PLEx $(n=21)$. Of the three, patients given AChEI only were liberated from mechanical ventilation (MV) earlier (6 days) compared with those in the AChEI + steroids (7 days) and PLEx ( $n=14$ days) groups, although the difference was not statistically significant. In terms of complication, two had asystole and four had pneumonia (20.8\% complication rate) in the AChEI only group; two had asystole and four had pneumonia (33.3\% complication rate) in the AChEI + steroids group; and five had asystole and five had pneumonia in the PLEx group (47.6\% complication rate). There were eight mortalities recorded, six due to cardiac arrhythmia and two from infection. Four out of the five who died of cardiac arrest received cholinergic drugs; however, signs of cholinergic excess, such 


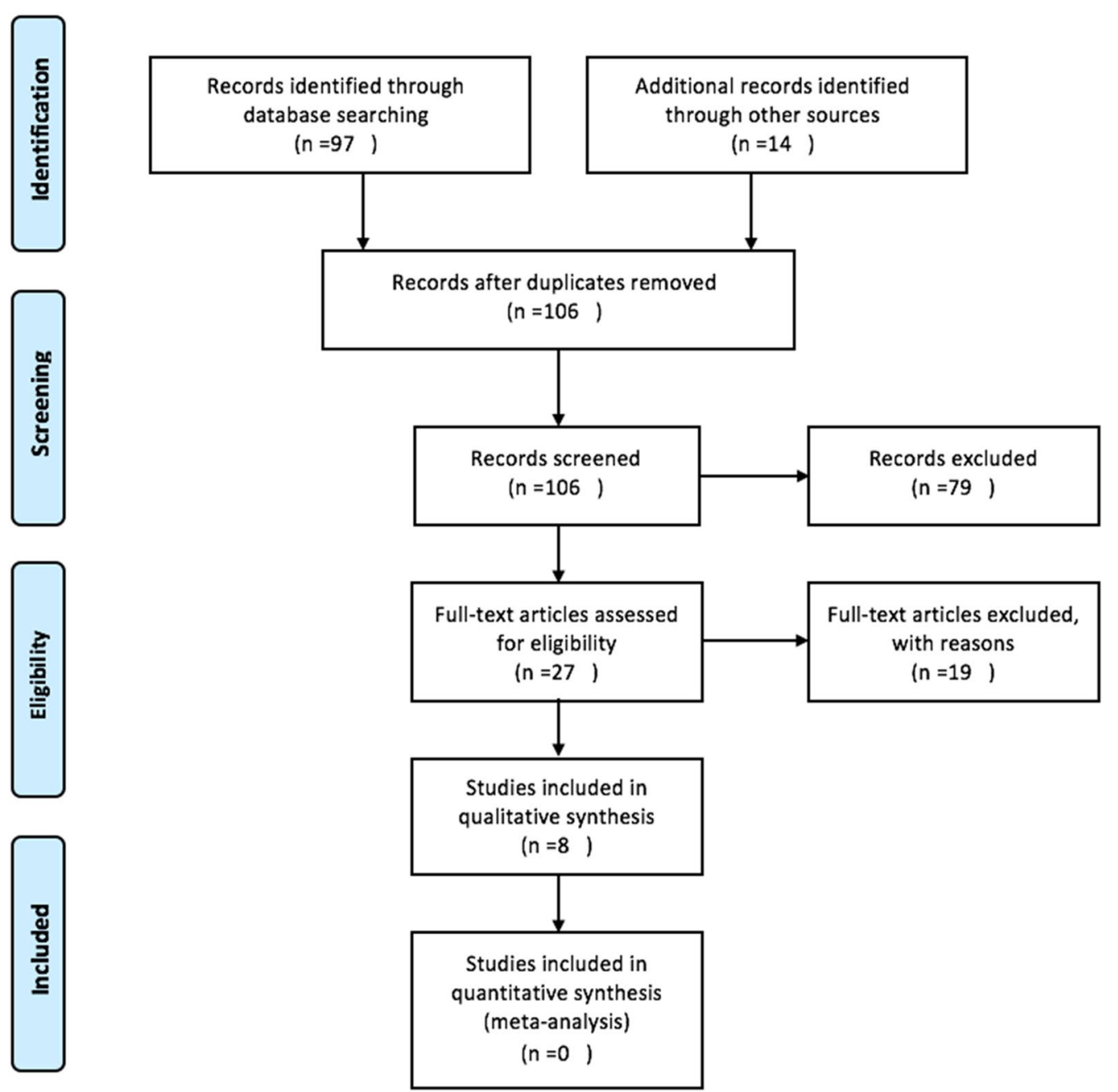

Fig. 1 Preferred Reporting Items for Systematic Reviews and Meta-analysis PRISMA flow diagram

as diarrhea, salivation, bradycardia, and abdominal cramping, were not observed prior to their demise. Although the authors concluded that these deaths may not be related to AChEI, caution when using AChEI during MC should always be observed. Selection bias was observed in this study, as patients with respiratory infection were placed in the "AChEI only group" because infection was a contraindication to PLEx at that time.

2. Thomas et al. (1997) [15] performed a single-center retrospective case review in 53 patients with $\mathrm{MC}$ who developed 73 episodes of MC. They discontinued pyridostigmine for all the patients and restarted the drug 1-2 days prior to extubation. All were given azathioprine, steroids, or plasmapheresis during MC. They assessed the pulmonary functions immediately prior to extubation and complications. The mean vital capacity increased from $21 \mathrm{ml} / \mathrm{kg} 3$ days post intubation to $27 \mathrm{ml} / \mathrm{kg}$ immediately prior to extubation. The mean peak expiratory flow and peak inspiratory flow immediately prior to extubation were 50 and $-43 \mathrm{~cm} \mathrm{H}_{2} \mathrm{O}$, respectively. Overall hospital mortality was $10 \%$, but there was no mention if the deaths were related to pyridostigmine.

3. Panda et al. (2004) [16] analyzed 11 patients who developed 12 episodes of MC out of 114 patients with MG. They discontinued the AChEI throughout the period of ventilation in all patients and continued the drug prior to discharge. All patients were given azathioprine steroids or PLEx. In these patients, $40 \%$ could walk unsupported and $70 \%$ could feed orally. There was no mention of complications specific to pyridostigmine. One of the most common precipitating factors for $\mathrm{MC}$ besides infection was withdrawal or noncompliance to AChEI. 
4. Sellman and Mayer (1985) [14] conducted a case series involving 17 patients who developed 20 episodes of MC, 11 of whom received neostigmine. The neostigmine dose was $1 \mathrm{mg}$ IM every $6 \mathrm{~h}$ administered after a trial of $1 \mathrm{mg}$ of edrophonium elicited no cholinergic side effects. Combining the AChEI alone and AChEI + prednisone groups $(n=11)$ yielded an average $\mathrm{MC}$ duration of 32.5 days and complication rate of 55\%. Compared with the PLEx + prednisone group $(n=7)$, the former group had worse MC duration and complication rates. However, unstable blood pressure, particularly hypotension, was one of the contraindications for PLEx. Although not mentioned, there was a high probability that the seven elderly patients with cardiac dysfunction, including fibrillation and arrhythmias, would not undergo PLEx, and most patients in this group were those with no, mild, or noncardiac complications only. It was established in the same study that those with medical problems had longer MC duration than those without (50 days vs. 18 days); hence, a selection bias may be prevalent. Nevertheless, the same study also recommended avoiding giving AChEI alone and concluded that PLEx in combination with prednisone was the most effective and safest among the groups. Because of the limited number of samples, this study lacked the power to determine any significant differences between groups.

5. Sharma et al. (2013) [17] conducted a prospective study of ten patients with MC from 2009 to 2010. They discontinued pyridostigmine and restarted prior to weaning. Despite being weaned off the ventilator, two of their patients died. One died of sudden cardiac arrhythmia, whereas the other one had thyrotoxicosis and cardiac arrest. Temporally, administration of pyridostigmine as a possible cause of these events cannot be excluded; nevertheless, the authors attributed these more to susceptibility of the heart muscle as another autoimmune target of MG.

6. Saltis et al. (1993) [18] described a 62-year-old man and a 21-year-old woman who were unresponsive to PLEx and in whom the dosage of steroids was increased during the $\mathrm{MC}$ period. In both cases, continuous IV infusion of pyridostigmine showed improvement of symptoms. In the first patient, the negative inspiratory flow increased from -35 to $-53 \mathrm{~cm} \mathrm{H}_{2} \mathrm{O}$ and forced vital capacity (FVC) increased from 0.75 to $2.4 \mathrm{l}$ in the first $7 \mathrm{~h}$ of infusion. In the second case, there was resolution of ptosis, and the patient was weaned from mechanical ventilation $36 \mathrm{~h}$ after the start of the infusion. Cholinergic side effects were also markedly decreased when pyridostigmine was switched from the IM route to infu- sion. They concluded that continuous administration of pyridostigmine is a potential alternative in the management of myasthenic exacerbations and may be particularly valuable in critical care situations.

7. Mishra et al. (2010) [13] reported a 27-year-old woman who had two episodes of MC post thymectomy, both triggered by sudden or inadequate intake of AChEI. In the first crisis, the patient was intubated after the primary physician decreased the existing AChEI dose. Oral pyridostigmine at a dose of $120 \mathrm{mg}$ every $6 \mathrm{~h}$ was given over 2 days with significant improvement. One week after discharge, the patient had another MC episode due to noncompliance to medication. Oral pyridostigmine at a similar dose was restarted and she was placed on intermittent noninvasive ventilation for respiratory support. No PLEx or IVIG was given. In this episode, the forced expiratory volume 1 (1.84 l), forced vital capacity FVC (1.92 l), PEF (4.32 l/s), and FEV1/FVC (95.8\%) improved. The authors concluded that a gradual increase in the dose of AChEI drugs and provision of intermittent non-invasive ventilation during the dosing interval was a safe and easy method of managing respiratory difficulties in patients with $\mathrm{MC}$ during the postoperative period. No cholinergic side effects were observed.

8. Briassoulis et al. (2000) [19] described a 10-year-old with postthymectomy MC who was administered continuous IV neostigmine after not responding to oral AChEI, prednisolone, and IVIG. On the first day of infusion, the negative inspiratory flow increased from -5 to $-35 \mathrm{~cm} \mathrm{H}_{2} \mathrm{O}$, and there was resolution of ptosis and marked improvement in respiratory strength. Weaning from mechanical ventilation started $36 \mathrm{~h}$ after reaching the maximum dose of infusion.

\section{Utility of AChEl in MC}

Two retrospective case studies and three case reports that utilized AChEI in the acute period of MC and three other observational studies that restarted the medication prior to weaning or extubation showed improvement in different outcome measures. Among those that utilized AChEI at the acute phase, one study showed that the outcome measures for continuous IV infusion of pyridostigmine alone had no significant difference when compared with combination of pyridostigmine and prednisone or PLEx alone [6]. Either pyridostigmine or neostigmine were given. Although several routes of administration were used, the continuous IV infusion of pyridostigmine at 1 to $2 \mathrm{mg} /$ hour was utilized in the study with the largest sample size [6]. 
The duration of MC was shortened whether the patients were on AChEI alone or AChEI in combination with recommended first-line treatments.

\section{Complications or Side Effects}

In two case reports, cholinergic side effects were prominent when the patients were started on oral and IM pyridostigmine $[20,21]$. However, when these were changed to continuous IV form, the cholinergic side effects disappeared. There was no documentation of occurrence of $\mathrm{CC}$ in all eight studies. Four out of five patients who died of cardiac arrhythmia received AChEI in one study [6]. However, these patients did not show any signs of cholinergic excess hence the authors concluded that these events were probably not AChEI related [6] Another study mentioned a complication rate as high as 55\%, mostly consisting of respiratory infections and cardiac dysrhythmia when patients were given AChEI with or without steroids [14]. Nevertheless, most patients given with AChEI in this study had prior comorbidities because infection and hemodynamic instability were contraindications for PLEx [14]. In another study in which two of the patients died of cardiac arrest, the authors attributed the incidents to susceptibility of the heart muscle as another autoimmune target of MG [17].

\section{Discussion}

Most recent guidelines recommend the use of PLEx or IVIG in combination with corticosteroids and discontinuation of AChEI in the management of MC [22]. Possible co-occurrence of $\mathrm{CC}$ and increase in bronchial secretions were cited for this recommendation [22]. However, although cholinergic side effects were observed in few studies, overt CC was not documented in any studies included in this systematic review. In addition, the high proportion of pneumonia in the cohort in which AChEI was given was probably secondary to noninclusion of patients whose trigger was infection in the PLEx group. Moreover, five studies concluded that continuous IV infusion of either neostigmine or pyridostigmine was probably safe and beneficial to patients with MC.

An AChEI holiday may increase the sensitivity of the postsynaptic membrane and may be of benefit when the drug is restarted prior to extubation according to MC guidelines [23]. However, in the present review, a benefit was observed whether the drug was given during or prior to weaning or extubation of patients with MC. In addition, one common cause of impending respiratory failure in developing countries was noncompliance; hence, holding the drug may push the patient to an $\mathrm{MC}$ state [17]. In one of the case reports, increasing the oral dose of pyridostigmine, in combination with intermittent noninvasive ventilation, shortened the $\mathrm{MC}$ duration to $36 \mathrm{~h}$ after the correction even in the absence of IVIG or PLEx [13].

The benefits of PLEx and IVIG have been proven in several studies [24]. In contrast, because of ethical concerns, there are no randomized controlled trials that determine the safety and efficacy of using AChEI in the management of MC. However, PLEx and IVIG are costly and need technical skills and machines for their administration, whereas AChEI is a cheap, readily available, and easy to administer drug, whether in IV, IM, or oral forms [1]. In developing countries and in countries with poor health care system, an alternative to IVIG or PLEx may be needed. This systematic review showed that adding AChEI may shorten the MC duration among patients with MG [6, 10, 13, 14, 19]. Nevertheless, most of the studies included have PLEx, IVIG, or immunomodulator as cointerventions that may act as confounders [10, $13,14,16,19]$. Nonetheless, in one study, the clinical outcome and complication for giving AChEI alone was not statistically different from giving PLEx. [6].

Cardiac dysrhythmia is one of the fatal complications possibly associated with AChEI during the MC period $[1,25]$. One case report even attributed hypercholinergic crisis to be a possible cause of vasospasm-induced myocardial infarction [26]. Nevertheless, the presence of several risk factors that predispose to cardiac complications, absence of parasympathetic symptoms suggestive of cholinergic overactivity, and no mention of AChEI dosage make the association less likely. Four out of five patients who received AChEI died of fatal arrhythmia in one of the studies included [6]; however, the absence of cholinergic symptoms prior to their demise led the authors to conclude that these events were probably not due to AChEI alone [6]. Takotsubo cardiomyopathy, giant cell myocarditis, and cardiac dysrhythmia were possible complications of thymomatous MG. Accordingly, high antistriational antibody titers may induce autoimmune attack to myocardium [27].

The limitation of this study is the high risk of bias inherent in observational studies. Because the assignment of exposure variable cannot be controlled as that of experimental studies, selection bias is unavoidable. Most patients with hemodynamic instability and sepsis were more likely to have AChEI alone because these were contraindications for PLEx. Unfortunately, these patients tended to have longer MC duration and higher complication and mortality rates whichever treatment group they were assigned.

Although the goal of this systematic review is to determine the efficacy and safety of AChEI alone to the management of $\mathrm{MC}$, cointerventions with high-dose steroids, IVIG, PLEx, and/or other immunomodulatory drugs 
in most studies confound its true effects. Hence, it was impossible to determine whether the benefit or the complication was from other treatment options or from AChEI alone. Nonetheless, because of the rarity of the condition, decision-making can only be based on systematic reviews supported by these types of studies at best.

\section{Future Recommendations}

A randomized controlled trial comparing continuous IV infusion of AChEI alone or in combination with steroids to IVIG or PLEx in patients with MC may give the best evidence on its use in this condition; however, current recommendations and guidelines prevent the use of AChEI in MC, and research proposals for such studies may have difficulty passing ethics standards. A systematic review of RCTs with meta-analysis can provide the best evidence for the use of AChEI in MC.

\section{Conclusions}

In places where IVIG or PLEx is not available, continuous IV infusion of pyridostigmine or neostigmine may be used with or without other cointerventions, provided that the patient is admitted in the intensive care unit and the airway has been secured adequately. Caution should be observed in using AChEI because of possible cardiac complications.

\begin{abstract}
Author details
${ }^{1}$ Department of Neurology, Graduate School of Medicine, Chiba University, 1-8-1 Inohana, Chuo-ku, Chiba 260-8670, Japan. ${ }^{2}$ Department of Epidemiology and Biostatistics, College of Public Health, University of the PhilippinesManila, Manila, Philippines. ${ }^{3}$ Section of Neurology, Department of the Neurosciences, Philippine General Hospital, University of the Philippines-Manila, Manila, Philippines.
\end{abstract}

\section{Acknowledgements}

Dr. Prado receives scholarship from Takeda Science Foundation.

\section{Author contributions}

MP designed the study. MP and KA collected the studies. MP and KA analyzed the data and wrote the manuscript. We also confirm that authorship requirements have been met, and the final manuscript has been approved by all authors.

\section{Sources of support}

None.

\section{Conflicts of interest}

The authors declare no conflicts of interest.

\section{Publisher's Note}

Springer Nature remains neutral with regard to jurisdictional claims in published maps and institutional affiliations.

Received: 22 January 2021 Accepted: 15 April 2021

Published online: 22 July 2021

\section{References}

1. Ahmed S, Kirmani JF, Janjua N, et al. An update on myasthenic crisis. Curr Treat Options Neurol. 2005;7(2):129-41. https://doi.org/10.1007/ s11940-005-0022-2.

2. Skeie GO, Apostolski S, Evoli A, et al. Guidelines for the treatment of autoimmune neuromuscular transmission disorders. Eur J Neurol. 2006;13(7):691-9. https://doi.org/10.1111/j.1468-1331.2006.01476.x

3. Kirmani JF, Yahia AM, Qureshi Al. Myasthenic crisis. Curr Treat Options Neurol. 2004:6:3-15.

4. Bershad EM, Feen ES, Suarez JI. Myasthenia gravis crisis. South Med J. 2008;101(1):63-9. https://doi.org/10.1097/SMJ.0b013e31815d4398.

5. Chaudhuri A, Behan PO. Myasthenic crisis. QJM. 2009;102(2):97-107. https://doi.org/10.1093/qjmed/hen152.

6. Berrouschot J, Baumann I, Kalischewski P, Sterker M, Schneider D. Therapy of myasthenic crisis. Crit Care Med. 1997;25(7):1228-35. https://doi.org/ 10.1097/00003246-199707000-00027.

7. Bedlack RS, Sanders DB. How to handle myasthenic crisis. Postgrad Med. 2000;107(4):211-22. https://doi.org/10.3810/pgm.2000.04.1003.

8. Goti P, Spinelli A, Marconi G, et al. Comparative effects of plasma exchange and pyridostigmine on respiratory muscle strength and breathing pattern in patients with myasthenia gravis. Thorax. 1995;50(10):1080-6. https://doi.org/10.1136/thx.50.10.1080.

9. Godoy DA, de Mello LJV, Masotti L, Di Napoli M. The myasthenic patient in crisis: an update of the management in neurointensive care unit. Arq Neuropsiquiatr. 2013;71(9A):627-39. https://doi.org/10.1590/0004-282X2 0130108.

10. Borel C. Management of myasthenic crisis. Crit Care Med. 1993;21(6):8212. https://doi.org/10.1097/00003246-199306000-00005.

11. Stroup DF, Berlin JA, Morton SC, et al. Meta-analysis of observational studies in epidemiology: a proposal for reporting. Meta-analysis of observational studies in epidemiology (MOOSE) group. JAMA. 2000;283(15):2008-12.

12. Sellman MS, Mayer RF. Treatment of Myasthenic Crisis in Late Life. South Med J. 1985;78(10):1208-10. https://doi.org/10.1097/00007611-19851 0000-00017.

13. Mishra SK, Krishnappa S, Bhat RR, Badhe A. Role of intermittent noninvasive ventilation in anticholinesterase dose adjustment for myasthenic crisis. Acta Anaesthesiol Taiwanica. 2010;48(1):53-4. https://doi.org/10. 1016/S1875-4597(10)60012-4.

14. Sellman MS, Mayer RF. Treatment of myasthenic crisis in late life. South Med J. 1985;78(10):1208-10. https://doi.org/10.1097/00007611-19851 0000-00017.

15. Thomas CE, Mayer SA, Gungor Y, et al. Myasthenic crisis: clinical features, mortality, complications, and risk factors for prolonged intubation. Neurology. 1997;48(5):1253-60. https://doi.org/10.1212/WNL.48.5.1253.

16. Panda S, Goyal V, Behari M, Singh S, Srivastava T. Myasthenic crisis: a retrospective study. Neurol India. 2004;52(4):453-6.

17. Sharma S, Lal V, Prabhakar S, Agarwal R. Clinical profile and outcome of myasthenic crisis in a tertiary care hospital: a prospective study. Ann Indian Acad Neurol. 2013;16(2):203-7. https://doi.org/10.4103/0972-2327. 112466.

18. Saltis LM, Martin BR, Traeger SM, Bonfliglio MF. Continuous infusion of pyridostigmine in the management of myasthenic crisis. Crit Care Med. 1993;21(6):938-40. https://doi.org/10.1097/00003246-199306000-00025.

19. Briassoulis G, Hatzis T, Liakopoulou T, Youroukos S. Continuous neostigmine infusion in post-thymectomy juvenile myasthenic crisis. J Child Neurol. 2000;15(11):747-9. https://doi.org/10.1177/088307380001501 106.

20. Saltis LM, Martin BR, Traeger SM, Bonfiglio MF. Continuous infusion of pyridostigmine in the management of myasthenic crisis. Crit Care Med. 1993;21(6):938-40. https://doi.org/10.1097/00003246-199306000-00025.

21. Briassoulis G, Hatzis T, Liakopoulou T, Youroukos S. Continuous neostigmine infusion in post- thymectomy juvenile myasthenic crisis. J Child Neurol. 2000;15:747-50. https://doi.org/10.1521/00332747.1963.11023 367.

22. Ciafaloni E. Myasthenia gravis and congenital myasthenic syndromes. Continuum (Minneap Minn). 2019;25(6):1767-84. https://doi.org/10. 1212/CON.0000000000000800.

23. Sonkar KK, Bhoi SK, Dubey D, Kalita J, Misra UK. Direct and indirect cost of myasthenia gravis: a prospective study from a tertiary care teaching 
hospital in India. J Clin Neurosci. 2017;38:114-7. https://doi.org/10.1016/j. jocn.2016.11.003.

24. Jani-Acsadi A, Lisak RP. Myasthenic crisis: guidelines for prevention and treatment. J Neurol Sci. 2007;261(1-2):127-33. https://doi.org/10.1016/j. jns.2007.04.045.

25. Lizarraga AA, Lizarraga KJ, Benatar M. Getting rid of weakness in the ICU: an updated approach to the acute management of myasthenia gravis and guillain-barré syndrome. Semin Neurol. 2016;36(6):615-24. https:// doi.org/10.1055/s-0036-1592106.
26. Comerci G, Buffon A, Biondi-Zoccai GGL, et al. Coronary vasospasm secondary to hypercholinergic crisis: an iatrogenic cause of acute myocardial infarction in myasthenia gravis. Int J Cardiol. 2005;103(3):335-7. https:// doi.org/10.1016/j.ijcard.2004.06.026.

27. Shivamurthy P, Parker MW. Cardiac manifestations of myasthenia gravis: a systematic review. IJC Metab Endocr. 2014;5:3-6. https://doi.org/10. 1016/j.ijcme.2014.08.003. 